\title{
Radiofrequency-targeted vertebral aug
versus traditional balloon kyphoplasty: radiographic and morphologic outcomes of an ex vivo biomechanical pilot study
}

\author{
This article was published in the following Dove Press journal: \\ Clinical Interventions in Aging \\ 17 November 2012 \\ Number of times this article has been viewed
}

\author{
Brian E Dalton' \\ Andrew C Kohm² \\ Larry E Miller ${ }^{3,4}$ \\ Jon E Block ${ }^{4}$ \\ Robert D Poser ${ }^{2}$ \\ 'Tri-State Neurological Surgeons, \\ Erie, PA, ${ }^{2}$ DFINE, Inc, San Jose, CA, \\ ${ }^{3}$ Miller Scientific Consulting, Inc, \\ Arden, NC, ${ }^{4}$ The Jon Block Group, \\ San Francisco, CA, USA
}

\begin{abstract}
Purpose: Traditional balloon kyphoplasty $(\mathrm{BK})$ is a common treatment for symptomatic vertebral compression fractures. The purpose of this study was to compare a novel vertebral augmentation technique, radiofrequency-targeted vertebral augmentation (RF-TVA), to BK for restoration of vertebral height, cavity creation, and polymethylmethacrylate (PMMA) delivery and interdigitation into the surrounding trabeculae.
\end{abstract}

Methods: This ex vivo biomechanical pilot study utilized 16 osteoporotic cadaveric vertebral bodies in a standardized fracture model to compare unipedicular RF-TVA $(n=8)$ to bipedicular BK $(n=8)$. Four specimens from each group were tested in loaded and unloaded conditions. All specimens were imaged, assessed for height restoration, and sectioned to observe PMMA distribution. A subset of specimens underwent computed tomography scanning to assess cavity creation and trabecular architecture prior to cement delivery.

Results: Anterior height restoration was greater with RF-TVA (median: 84\%, interquartile range: $62 \%-95 \%$ ) compared to BK (median: 69\%, interquartile range: $60 \%-81 \%$ ), although the difference did not achieve statistical significance $(P=0.16)$. Anterior height restoration was numerically greater under loaded (median: $70 \%$ versus 66\%) and unloaded (median: $94 \%$ versus 77\%) conditions with RF-TVA versus BK. RF-TVA produced more discrete cavities and less native trabecular destruction compared to marked trabecular destruction observed with $\mathrm{BK}$. RF-TVA consistently showed a well-identified focal area of PMMA with an extensive peripheral zone of PMMA interdigitation, providing mechanical interlock into the adjacent intact trabecular matrix. In contrast, BK yielded little evidence of PMMA interdigitation beyond the boundaries created by the balloon tamp due to the crushed trabecular bone peripherally.

Conclusion: RF-TVA achieves favorable vertebral height restoration with targeted PMMA delivery and less trabecular destruction compared to BK. RF-TVA has potential clinical utility in the aging population with painful osteoporotic vertebral fracture.

Keywords: balloon kyphoplasty, fracture, polymethylmethacrylate, radiofrequency targeted, vertebral augmentation

\section{Introduction}

Vertebral compression fracture (VCF) is a common injury in the elderly with an incidence of 700,000 per year in the United States alone. ${ }^{1}$ Conservative treatment of painful VCFs has only modest short-term effectiveness and is associated with poor long-term outcomes. ${ }^{2}$ Percutaneous balloon kyphoplasty (BK) is widely used to treat VCFs and has demonstrated clinically meaningful symptom improvement in most,
Correspondence: Robert Poser 3047 Orchard Parkway, San Jose, CA 95134 , USA

Tel + | 408770 | I 20

Fax +I 4085834314

Email bobp@dfineinc.com 
but not all, studies. ${ }^{3}$ However, the effectiveness of BK is, in part, dependent on the handling characteristics, delivery methods, and curing properties of the injected polymethylmethacrylate (PMMA) bone cement used to reduce and stabilize the fracture construct. ${ }^{4-7}$

Adverse events related to PMMA use are infrequent, but may be due to thermal necrosis, volumetric PMMA shrinkage, and increased stiffness of the composite compared to normal bone. ${ }^{4-9}$ Percutaneous introduction of low-viscosity PMMA into the vertebral body, as with the traditional BK procedure, may not fully support the initial fracture reduction..$^{8,9}$ Vertebral expansion, cavity creation, and height restoration utilizing a BK balloon tamp crushes peripheral noninvolved intravertebral trabecular matrices prior to PMMA filling, and may place the treated vertebra at risk for structural damage and refracture. ${ }^{8-10}$ While tamping of the crushed trabecular structures may prevent PMMA extravasation, ${ }^{11}$ it may have the untoward effect of hindering PMMA interdigitation, creating a zone of necrotic bone surrounding the injected bone cement bolus. ${ }^{12}$ Subsequent resorption of necrotic bone may cause PMMA loosening, PMMA mobility, adverse intravertebral stress, and an increased risk of fracture across the remaining trabecular bone. ${ }^{13}$

Radiofrequency-targeted vertebral augmentation (RFTVA) is a percutaneous vertebral augmentation technique that was developed to address some of the limitations of BK. This technique involves the use of a navigational osteotome to create a targeted intravertebral cavity, thereby preserving the noninvolved and structurally sound native vertebral body trabeculae, followed by introduction of an ultrahigh-viscosity PMMA with extended working time due to concurrent RF heating. Continuous controlled distribution of ultrahigh-viscosity PMMA increases the ability to stabilize the fracture and, subsequently, to mechanically interlock the PMMA with the intact trabeculae. With further delivery under fluoroscopy, controlled vertebral height restoration can be achieved. ${ }^{14-16}$ The purpose of this ex vivo biomechanical pilot study was to compare RF-TVA to traditional $\mathrm{BK}$ for restoration of vertebral height, cavity creation, and PMMA delivery and interdigitation into the surrounding trabeculae.

\section{Methods}

\section{Vertebral body preparation}

A cadaveric model protocol to approximate the vertebral body tissue encountered with in vivo conditions was used. Fresh frozen human vertebral specimens were maintained at $-20^{\circ} \mathrm{C}$ until approximately 24 hours prior to testing.
Sixteen vertebral specimens (eight per treatment group) from six human spine specimens (T9-T12) were thawed to room temperature and all residual soft tissue was removed via careful manual dissection. All vertebral bodies were screened for gross anatomical defects. Dual-energy X-ray absorptiometry was performed to measure bone mineral density. All specimens were confirmed as osteoporotic, defined as a T-score less than -2.5 compared to age-matched norms. Vertebral bodies were prepared by transecting the pedicles and removing all disc material from the endplates. The caudal and cephalad surfaces of each vertebral body were rigidly embedded in a urethane potting compound (Smooth-On, Inc, Easton, PA) and each composite was positioned with the centroid located in the middle of the potting fixture.

\section{VCF creation}

Prepared vertebral bodies were mounted in individual custom semiconstrained fixtures and rigidly attached to a servohydraulic load frame (8521S; Instron, Norwood, MA) in order to create consistent VCFs. All specimens were tested in air and preconditioned for ten cycles loaded between $-500 \mathrm{~N}$ and $-200 \mathrm{~N}$ of compression at a test frequency of $1 \mathrm{~Hz}$. Immediately following preconditioning, loads were applied at a displacement rate of $5 \mathrm{~mm} /$ minute with a data acquisition rate of $20 \mathrm{~Hz}$. Monotonic testing was performed until the anterior height of the vertebral body was reduced by $30 \%$ compared to the measured baseline height (Figure 1). Failure loads during VCF creation for each specimen group were recorded.

\section{Loading conditions}

Each procedure was performed in four vertebrae under a $150 \mathrm{~N}$ follower load and in four vertebrae under no load. A follower load was used to increase the clinical relevance of this model by applying a load to the vertebral body physiologically representative of a patient in the supine position. ${ }^{17}$ The follower load testing technique applies a compressive load along the path that approximates the tangent of the lumbar spine curvature and models resistance (due to fracture consolidation that occurs within the early weeks postfracture) that the force applied by the augmentation procedure to restore height must overcome.

\section{Procedures}

All procedures were performed using fluoroscopic guidance by the same fellowship-trained neurosurgeon with extensive experience in the performance of RF-TVA and BK for treatment of VCFs. 

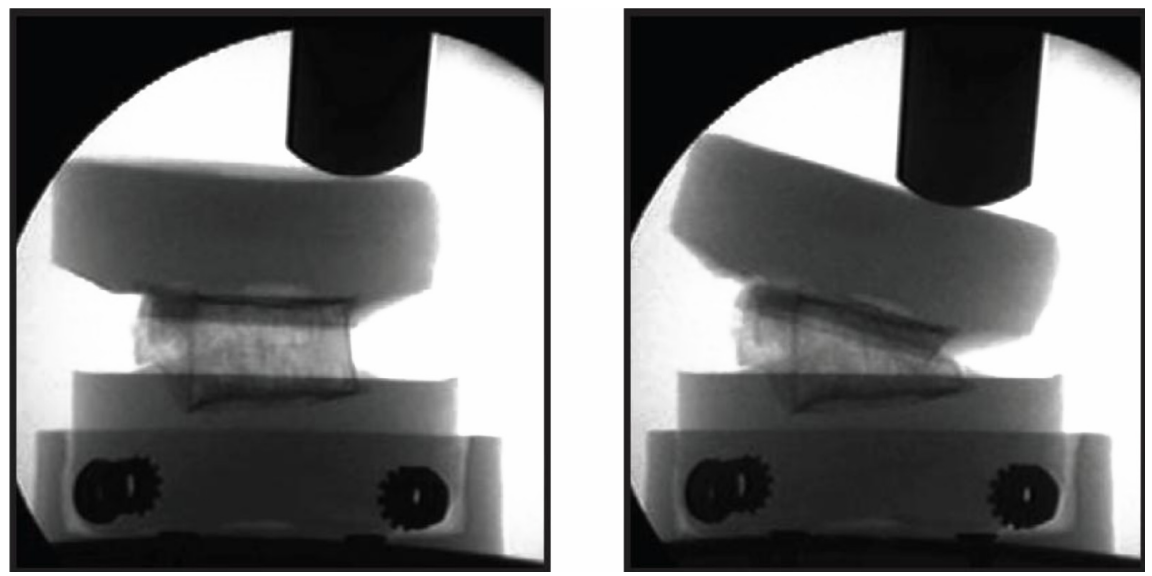

Figure I Vertebral specimen within loading frame before (left) and after (right) application of displacement load for creation of vertebral compression fracture.

RF-TVA was performed via a unipedicular approach with site-specific cavity creation followed by remotely controlled delivery of ultrahigh-viscosity cement (StabiliT ${ }^{\circledR}$ ER $^{2}$ Bone Cement; DFINE, Inc, San Jose, CA) using the StabiliT Vertebral Augmentation System (DFINE) (Figure 2). After placement of a working cannula, an initial cavity was created by inserting a straight coring osteotome (VertecoR ${ }^{\circledR}$ StraightLine Osteotome; DFINE) that permits biopsy. A navigational osteotome (VertecoR MidLine Osteotome; DFINE) was then inserted through the working cannula. The MidLine Osteotome was navigated through the vertebral body by rotating the handle to articulate the distal beveled tip as the osteotome was advanced through the vertebral body to the anterior third and across the midline in a controlled fashion. The MidLine Osteotome was then reoriented and additional passes made to create site-specific cavities (Figure 3). This resulted in well-defined cavities with minimal disruption of adjacent trabeculae that served as preferential paths for cement delivery. The PMMA, which has an extended working time compared to standard PMMA, was then converted

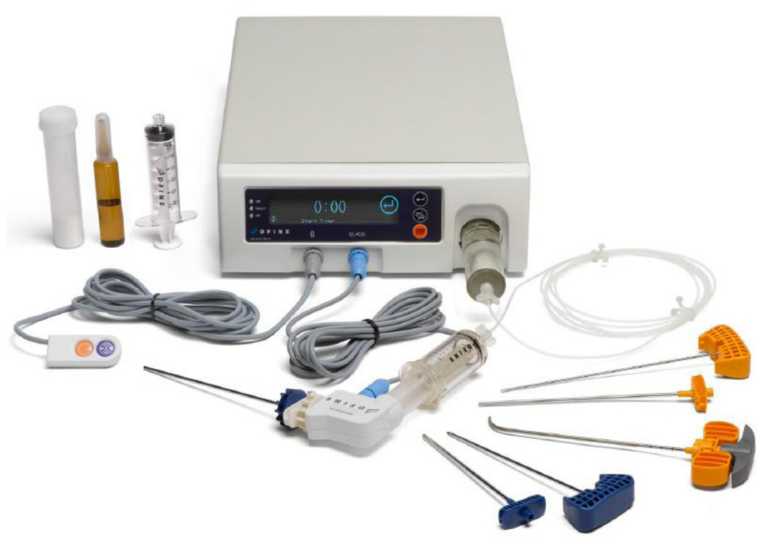

Figure 2 StabiliT ${ }^{\circledR}$ Vertebral Augmentation System (DFINE, Inc, San Jose, CA). to an ultrahigh-viscosity PMMA as it passed through the Activation Element (where it was heated by application of radiofrequency) immediately prior to entering the delivery cannula. The ultrahigh-viscosity PMMA was delivered at a continuous rate of $1.3 \mathrm{cc} /$ minute using a remote-controlled automated hydraulic delivery system. Intermittently during PMMA delivery, intravertebral filling was fluoroscopically monitored and terminated when the treating physician deemed that the fill was adequate.

Bipedicular BK was performed using standard techniques described previously (KyphX ${ }^{\circledR}$ Osteo Introducer ${ }^{\circledR}$ System; Medtronic, Inc, Minneapolis, MN) and included insertion of an inflatable bone tamp (KyphX Xpander ${ }^{\circledR}$ Inflatable Bone Tamp; Medtronic), balloon inflation for vertebral cavity creation, height restoration, and filling with PMMA (KyphX HV-R ${ }^{\mathrm{TM}}$ High-Viscosity Radiopaque Bone Cement; Medtronic) ${ }^{18}$

\section{Radiographic assessment}

Prior to preconditioning and immediately following the monotonic loading test, radiographs were acquired on all specimens for vertebral body height comparisons.
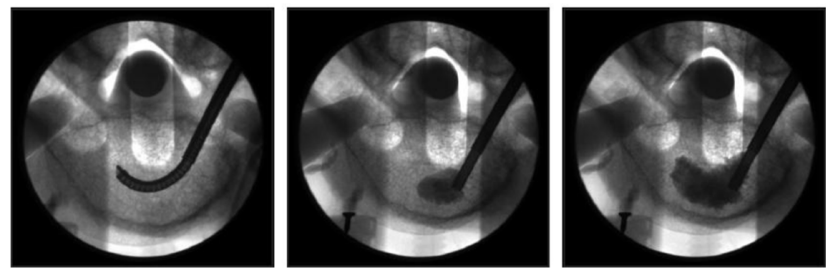

Figure 3 Stepwise unipedicular radiofrequency-targeted vertebral augmentation procedure including controlled intravertebral cavity creation with MidLine Osteotome (DFINE, Inc, San Jose, CA) (left), targeted delivery of StabiliT ${ }^{\circledR}$ ER $^{2}$ (DFINE) ultrahigh-viscosity polymethylmethacrylate beginning anteriorly (middle), and continued delivery of polymethylmethacrylate with trabecular interdigitation and controlled vertebral height restoration (right). 
Following cavity creation and prior to cement delivery, two vertebrae in each treatment group were scanned using computed tomography (slice thickness $0.625 \mathrm{~mm}$; LightSpeed16; GE Healthcare, Little Chalfont, United Kingdom) to evaluate location and shape of the cavity as well as adjacent trabecular morphology. Following cement delivery, radiographic and computed tomography images were acquired and vertebral height was measured prior to serial sectioning. Anterior vertebral height was measured using the technique described by McKiernan et al. ${ }^{19}$ Vertebral height was defined as the distance between points on the most anterior aspects of the superior endplate and the inferior endplate. All prefracture, postfracture, and postvertebral augmentation images for each vertebra were read by five independent reviewers with the average of the readings used for data analysis. Height restored (anterior measure posttreatment minus post fracture) and height loss (anterior measure intact vertebra minus postfracture) were used to calculate percent height restoration (height restored/height loss $\times 100$ ).

\section{PMMA interdigitation and trabecular assessment}

Following sectioning, specimens were visually examined for location and interdigitation of PMMA. Excellent PMMA interdigitation was identified when PMMA was interspersed throughout the trabeculae. Poor PMMA interdigitation was identified by a solid PMMA mass due to PMMA lumping without interspersion. Dissolution of PMMA was performed by soaking the sectioned specimens in acetone prior to examination of the remaining trabecular structures.

\section{Data analysis}

Data were reported as mean \pm standard deviation or median (interquartile range), depending on normality assumptions. Comparison of vertebral height restoration between groups was performed using the Wilcoxon signed-rank test.

\section{Results}

Vertebral specimens had an average age at death of $86 \pm 9$ years (range: 72-96 years). Bone mineral density averaged $0.64 \pm 0.15 \mathrm{~g} / \mathrm{cm}^{2}$ (range: $0.39-0.76 \mathrm{~g} / \mathrm{cm}^{2}$ ), with all specimens meeting the criterion for osteoporosis. Mean maximum failure load was similar $(P=0.18)$ for RF-TVA $(1544 \pm 526 \mathrm{~N})$ and BK $(1979 \pm 484 \mathrm{~N})$.

Anterior height restoration was greater with RF-TVA (median: 84\%, interquartile range: 62\%-95\%) compared to BK (median: 69\%, interquartile range: 60\%-81\%), although the difference did not achieve statistical significance
$(P=0.16)$. When analyzed according to loading condition, anterior height restoration was numerically greater with RF-TVA under loaded (median: 70\% versus 66\%) and unloaded (median: 94\% versus 77\%) conditions, although the small sample precluded formal statistical analysis in this subgroup.

Inspection of computed tomography images revealed the creation of distinct cavities prior to cement delivery with both procedures. However, RF-TVA resulted in more discrete cavities leading to less native trabecular destruction compared to the marked trabecular destruction observed with BK (Figure 4).

Mean PMMA volume was $6.6 \pm 1.1 \mathrm{cc}$ for RF-TVA and $6.5 \pm 1.1 \mathrm{cc}$ for BK. Following serial sectioning and prior to PMMA dissolution, specimens subjected to RF-TVA consistently showed a well-identified focal area of PMMA with an extensive peripheral zone of PMMA interdigitation and mechanical interlock into the adjacent trabecular matrix (Figure 5). In contrast, specimens in the BK group demonstrated little evidence of PMMA interdigitation beyond the boundaries created by the balloon tamp due to the peripheral crushed trabecular bone. Following PMMA dissolution, increased trabecular bone loss due to balloon inflation was confirmed in these specimens (Figure 6).

\section{Discussion}

This ex vivo biomechanical pilot study showed that RF-TVA achieves favorable vertebral height restoration with focused PMMA delivery and less trabecular destruction compared to BK. The novel aspects of RF-TVA include a unipedicular approach and use of an articulating navigational osteotome that, unlike BK, allows targeted creation of a site-specific, well-defined, minimally destructive intravertebral cavity as well as controlled delivery of an ultrahigh-viscosity cement
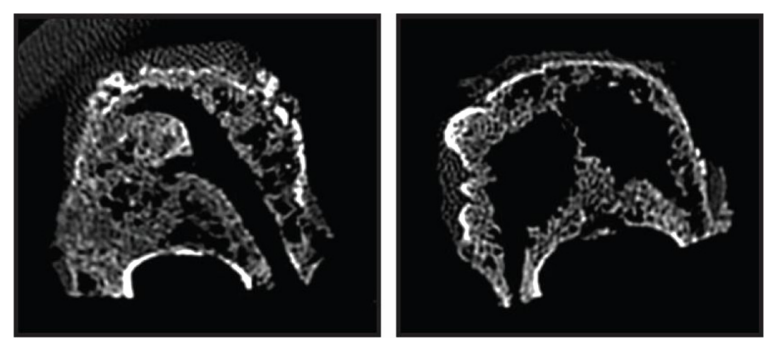

Figure 4 Computed tomography images of vertebrae following cavity creation, prior to cement augmentation.

Notes: Discrete directional cavity created using the navigational MidLine Osteotome (DFINE, Inc, San Jose, CA) via a unipedicular approach during radiofrequency-targeted vertebral augmentation preserves more trabecular bone and creates preferential path(s) for targeted flow of ultrahigh-viscosity cement (left). Large cavities resulting from bipedicular balloon expansion reduce the amount of trabecular bone and area for cement interdigitation (right). 

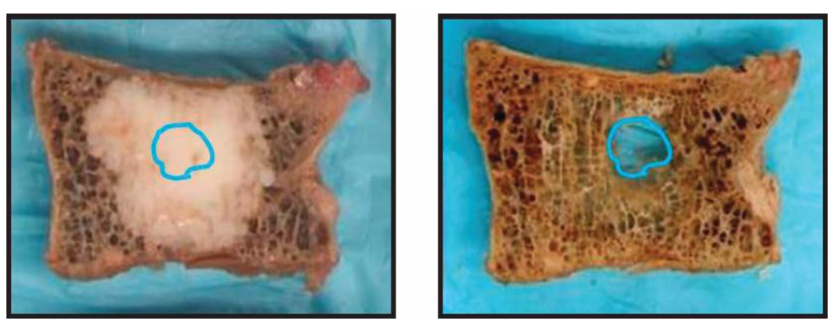

Figure 5 Radiofrequency-targeted vertebral augmentation sectioned specimen demonstrates a greater depth of cement interdigitation beyond the surgically created cavity (left) and less trauma to native trabeculae and a cavity that consumes a smaller percentage of the vertebral body after polymethylmethacrylate dissolution (right).

resulting in optimal void filling and extensive interdigitation into the adjacent trabecular bone.

This study found that a significant percentage of anterior vertebral height restoration can be achieved utilizing RF-TVA and BK under clinically relevant loading conditions. Despite less anterior vertebral height restoration in both groups under loaded versus unloaded conditions, there was greater height restoration with RF-TVA with unloaded conditions. This phenomenon was likely caused by the targeted delivery and interdigitation of ultrahigh-viscosity RF-heated cement into the remaining trabeculae. Under loaded conditions, which more realistically simulate in vivo conditions, anterior vertebral height restoration was significant in both groups.

Targeted cavity creation was achieved with minimal sacrifice of the adjacent trabecular matrix; however, cavities created using bipedicular BK sacrificed the trabecular matrix, were largely dictated by balloon geometry, and were often limited to the axial orientation of the working cannula through which the balloons were inserted. The cavity created with BK inconsistently included all areas of trabecular compromise. Additionally, upon filling, PMMA interdigitation and mechanical interlock of the PMMA with the adjacent trabecular matrix was poor. Tamped trabecular bone following PMMA filling has been shown to be necrotic with the possibility of resorption and potential for PMMA loosening and mobilization. ${ }^{12}$ Using a finite element model, Dabirrahmani et al reported that under these PMMA-bone interface parameters, and with normal load conditions, the PMMA may become loose and dislocate from the original cavity position. ${ }^{13}$

The penetration of standard PMMA into trabecular bone is dependent on the porosity of the available trabecular matrix, the viscosity of the introduced PMMA, and the stability of the PMMA during curing., ${ }^{5,7,13,20-25}$ Since PMMA has no adhesive properties, its primary function is as a filler, which underscores the importance of trabecular interlock. Penetration of PMMA into surrounding intact trabecular matrices can have a significant effect on the mechanical integrity of the overall bone-PMMA construct including the dampening of increased stress within the vertebral body, minimization of stress shielding by better load distribution, and maintenance of intravertebral bone and bone density. ${ }^{4,21-23,25}$ In benchtop studies of the bone-PMMA interface, Graham et al reported that bone porosity was the leading factor in the fracture resistance of the bone-PMMA interface. ${ }^{21}$ Peripheral interdigitation of PMMA maintains mechanical integrity of the construct in compression resulting in a more uniform load transfer between the vertebral endplates and vertebral body. ${ }^{13}$ The PMMA delivery in RFTVA may, therefore, aid in initially stabilizing the fracture and, upon bone-to-PMMA mechanical interlock, minimizing the potential additive risks of bone necrosis and resorption, interface fibrous membrane formation, and PMMA loosening and mobilization. The utility of an articulating cavity creation device to achieve remote access within the vertebral body and reproducible targeted cavity creation via a unipedicular approach is further underscored by Chung et al, who reported that placement of a unipedicular bone
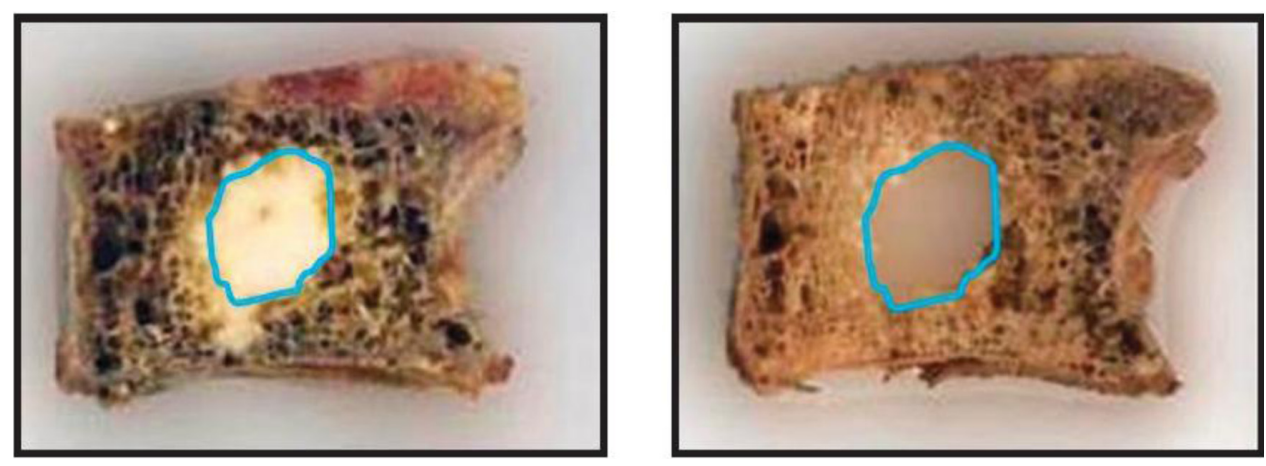

Figure 6 Balloon kyphoplasty sectioned specimen demonstrates notably less interdigitation of bone cement outside the larger surgically created cavity (left) and increased loss of peripheral trabecular bone after polymethylmethacrylate dissolution (right). 
tamp more centrally within the vertebral body violated the medial wall of the pedicle resulting in PMMA leakage into the spinal canal. ${ }^{26}$

A limitation of the current study includes the use of prepared cadaveric vertebral bodies since ex vivo models cannot reproduce the exact in vivo environment encountered during surgery. However, the use of osteoporotic bone specimens allowed the researchers to replicate and treat trabecular involvement similar to in vivo conditions following VCF. The application of a load during treatment of a subset of these specimens provided further clinical relevance to the study findings. A second limitation of this study was the small sample size, which limited the statistical power of anterior vertebral height restoration outcome comparisons. However, the sectioned histological review of PMMA distribution showed superior cement interdigitation and mechanical interlock in all specimens treated with RF-TVA. Despite these limitations, these results provide mechanistic insight to outcomes of a human clinical trial that demonstrated shorter procedural time, reduced leakage rates, and equivalent height restoration with RF-TVA compared to $\mathrm{BK} .{ }^{16}$

\section{Conclusion}

In conclusion, unipedicular RF-TVA and bipedicular BK achieve satisfactory vertebral height restoration in an ex vivo VCF model. However, RF-TVA produces less trabecular destruction and superior PMMA interdigitation compared to BK. RF-TVA has potential clinical utility in the aging population with painful osteoporotic VCFs.

\section{Acknowledgments}

The authors thank Mr Randy Asher for graphical assistance. This study was supported by DFINE, Inc (San Jose, CA).

\section{Disclosure}

AWK and RDP are employees of DFINE, Inc.

\section{References}

1. Samelson EJ, Hannan MT. Epidemiology of osteoporosis. Curr Rheumatol Rep. 2006;8(1):76-83.

2. Lyritis GP, Mayasis B, Tsakalakos N, et al. The natural history of the osteoporotic vertebral fracture. Clin Rheumatol. 1989;8(Suppl 2): 66-69.

3. Robinson Y, Heyde CE, Forsth P, Olerud C. Kyphoplasty in osteoporotic vertebral compression fractures - guidelines and technical considerations. J Orthop Surg Res. 2011;6:43.

4. Boger A, Bisig A, Bohner M, Heini P, Schneider E. Variation of the mechanical properties of PMMA to suit osteoporotic cancellous bone. J Biomater Sci Polym Ed. 2008;19(9):1125-1142.
5. Boger A, Wheeler K, Montali A, Gruskin E. NMP-modified PMMA bone cement with adapted mechanical and hardening properties for the use in cancellous bone augmentation. J Biomed Mater Res B Appl Biomater. 2009;90(2):760-766.

6. Verlaan JJ, Oner FC, Slootweg PJ, Verbout AJ, Dhert WJ. Histologic changes after vertebroplasty. J Bone Joint Surg Am. 2004;86-A(6): $1230-1238$.

7. Webb JC, Spencer RF. The role of polymethylmethacrylate bone cement in modern orthopaedic surgery. J Bone Joint Surg Br. 2007;89(7): 851-857.

8. Fribourg D, Tang C, Sra P, Delamarter R, Bae H. Incidence of subsequent vertebral fracture after kyphoplasty. Spine (Phila Pa 1976). 2004;29(20):2270-2276.

9. Kim MJ, Lindsey DP, Hannibal M, Alamin TF. Vertebroplasty versus kyphoplasty: biomechanical behavior under repetitive loading conditions. Spine (Phila Pa 1976). 2006;31(18):2079-2084.

10. Machinis TG, Fountas KN, Feltes CH, Johnston KW, Robinson JS. Pain outcome and vertebral body height restoration in patients undergoing kyphoplasty. South Med J. 2006;99(5):457-460.

11. Phillips FM, Todd Wetzel F, Lieberman I, Campbell-Hupp M. An in vivo comparison of the potential for extravertebral cement leak after vertebroplasty and kyphoplasty. Spine (Phila Pa 1976). 2002; 27(19):2173-2178

12. Togawa D, Kovacic JJ, Bauer TW, Reinhardt MK, Brodke DS, Lieberman IH. Radiographic and histologic findings of vertebral augmentation using polymethylmethacrylate in the primate spine: percutaneous vertebroplasty versus kyphoplasty. Spine (Phila Pa 1976). 2006;31(1):E4-E10.

13. Dabirrahmani D, Becker S, Hogg M, Appleyard R, Baroud G, Gillies M. Mechanical variables affecting balloon kyphoplasty outcome - a finite element study. Comput Methods Biomech Biomed Engin. 2012;15(3): 211-220.

14. Miller MA, Race A, Gupta S, Higham P, Clarke MT, Mann KA. The role of cement viscosity on cement-bone apposition and strength: an in vitro model with medullary bleeding. J Arthroplasty. 2007;22(1): 109-116.

15. Ruger M, Schmoelz W. Vertebroplasty with high-viscosity polymethylmethacrylate cement facilitates vertebral body restoration in vitro. Spine (Phila Pa 1976). 2009;34(24):2619-2625.

16. Pflugmacher R, Bornemann R, Koch EM, et al. Comparison of clinical and radiological data in the treatment of patients with osteoporotic vertebral compression fractures using radiofrequency kyphoplasty or balloon kyphoplasty. Z Orthop Unfall. 2012;150(1):56-61. German.

17. Sato K, Kikuchi S, Yonezawa T. In vivo intradiscal pressure measurement in healthy individuals and in patients with ongoing back problems. Spine (Phila Pa 1976). 1999;24(23):2468-2474.

18. Peh WC, Munk PL, Rashid F, Gilula LA. Percutaneous vertebral augmentation: vertebroplasty, kyphoplasty and skyphoplasty. Radiol Clin North Am. 2008;46(3):611-635.

19. McKiernan F, Faciszewski T, Jensen R. Reporting height restoration in vertebral compression fractures. Spine (Phila Pa 1976). 2003; 28(22):2517-2521

20. Breusch SJ, Norman TL, Schneider U, Reitzel T, Blaha JD, Lukoschek M. Lavage technique in total hip arthroplasty: jet lavage produces better cement penetration than syringe lavage in the proximal femur. J Arthroplasty. 2000;15(7):921-927.

21. Graham J, Ries M, Pruitt L. Effect of bone porosity on the mechanical integrity of the bone-cement interface. J Bone Joint Surg Am. 2003;85-A(10):1901-1908.

22. Krause WR, Krug W, Miller J. Strength of the cement-bone interface. Clin Orthop Relat Res. 1982;(163):290-299.

23. Mann KA, Ayers DC, Werner FW, Nicoletta RJ, Fortino MD. Tensile strength of the cement-bone interface depends on the amount of bone interdigitated with PMMA cement. J Biomech. 1997;30(4): 339-346. 
24. Rey RM Jr, Paiement GD, McGann WM, et al. A study of intrusion characteristics of low viscosity cement Simplex-P and Palacos cements in a bovine cancellous bone model. Clin Orthop Relat Res. 1987;(215): 272-278.

25. Walker PS, Soudry M, Ewald FC, McVickar H. Control of cement penetration in total knee arthroplasty. Clin Orthop Relat Res. 1984; (185):155-164.
26. Chung HJ, Chung KJ, Yoon HS, Kwon IH. Comparative study of balloon kyphoplasty with unilateral versus bilateral approach in osteoporotic vertebral compression fractures. Int Orthop. 2008;32(6): 817-820.

\section{Publish your work in this journal}

Clinical Interventions in Aging is an international, peer-reviewed journal focusing on evidence-based reports on the value or lack thereof of treatments intended to prevent or delay the onset of maladaptive correlates of aging in human beings. This journal is indexed on PubMed Central, MedLine, the American Chemical Society's 'Chemical Abstracts

\section{Dovepress}

Service' (CAS), Scopus and the Elsevier Bibliographic databases. The manuscript management system is completely online and includes a very quick and fair peer-review system, which is all easy to use. Visit $\mathrm{http}: / /$ www.dovepress.com/testimonials.php to read real quotes from published authors.

Submit your manuscript here: http://www.dovepress.com/clinical-interventions-in-aging-journal 